EPiC Series in Engineering
Volume 3, 2018, Pages 2333-2341
HIC 2018. 13th International
Conference on Hydroinformatics

\title{
Capitalizing RFID technology as cost-effective real-time process monitoring tool in wastewater treatment: Two case studies
}

\author{
Wouters, J.W ${ }^{1,2}$,Seelam, J.S and Agema, K.J ${ }^{3}$ \\ ${ }^{1}$ BW Products BV \\ ${ }^{2}$ Brightwork BV \\ ${ }^{3}$ Wetterskip Fryslân \\ Corresponding author: h.wouters@brightwork.nl
}

\begin{abstract}
The legislative norms for treated wastewater diffuse in terms of nitrogen $(\mathrm{N})$ and phosphorus $(\mathrm{P})$ concentrations are becoming increasingly stringent in the EU region. Compliance with the consent values compelled the water authorities to implement moving bed biofilters (MBFs) for tertiary stage effluent polishing. However, on-site and field surveys reveal that numerous MBF units suffer from non-optimal operational conditions, logistical challenges and irregular monitoring. This makes meeting the N-P criterion quite a challenging and expensive affair. It is therefore important to optimize their day-to-day operations, facilitate access to reliable and real-time status updates, and troubleshoot the failures. In this direction, an "internet-of-things", radio frequency ID (RFID) and cloud based monitoring and control tool, Sand-Cycle, was successfully developed, tested and implemented to monitor MBFs. The current study presents fullscale application of the developed remote control and mote technology at two wastewater treatment works. Sand-Cycle illustrated real-time dashboards indicating performance grading factors viz. in-situ average sand circulation rate, active bed volume and filter homogeneity. This presented clear instructions for detected malfunctions and enabled the operators to optimize the MBF output with limited effort. Further technical and technological advancements of such IoT based setups can actively assist in tackling long-term sustainability and wastewater management issues.
\end{abstract}

Keywords: data analytics, real-time monitoring, moving bed biofilters, remote control, RFID, Sand-Cycle 


\section{Introduction}

The practical applicability of Radio Frequency IDentification (RFID) as an identification mechanism dates back to the years of the second world war (1939-1945) to differentiate Friend or Foe (IFF) military aircrafts (Leena Kumari, 2015)]. The working principle includes an identification process which is activated by wireless communication between a tag attached to an entity and an antenna via radiofrequency waves. The wi-fi transmission is translated into data points which is further analysed to map the activity/identity of the entity. At present, RFID technology found over 3,000 applications and has been heavily used for logistics, supply chain management and quick response systems (F. Bibi, 2017). Active academic and technical research aimed at construction, data security, reliability of the tags and economics is being performed to further diversify its functionality (X. Zhu, 2012). Such techno-academic advancements have opened up several avenues for active collaboration with data analytics and big data management to extend its service from simple inventory tracking to other utilities. Water and wastewater industry offers to be the latest and promising domain for RFID utility expansion. The EU guidelines laid by Water Framework Directive (WFD) also assert the necessity to include tools for effective data management in order to achieve reliable wastewater treatment performance (The EU Water Framework Directive, 2010). Such tools can ensure efficient and reliable plant performance thereby improving water quality and reducing operational and maintenance costs. Specially, use of passive LF RFID tags operating at low frequency $(125-134 \mathrm{kHz})$ in (waste)water systems is a favorable option as they show minimal interference with metals and liquids (F. Bibi, 2017). These tags are characterized by their battery-less construction, lower power consumption and their penetration ability through non-metal objects within one foot range. They typically obtain energy from the signal transmitted by the antenna making them maintenance-free and user-friendly.

As a pioneering approach, RFID-based smart monitoring tool, Sand-Cycle (www.sandcycle.com), using passive LF tags, internet-of-things and cloud-based storage platform was introduced into wastewater handling (SandCycle, 2017). Continuous moving bed biofilters (MBFs) were chosen as an initial reference among the available wastewater treatment technologies for developing and testing the integrated RFID framework. MBFs are single reactor based wastewater effluent polishing systems implemented for simultaneous N-P removal in drinking water, domestic wastewater and industrial (paper and pulp, chemical, steel, food, mining and mineral) wastewater treatment facilities (J.P., J.W., M.P., \& J.M., 2000). They work on the principle of (bio)filtration, uninterrupted and homogeneous sand circulation of a filter bed with help of an airlift to remove pollutants (Fig.1). The filtration process occurs through retaining of pollutants within the pores of the filter bed and possible biological degradation (nitrification/denitrification). The filter bed constantly moves downwards with fixed rate (typically $0.3-0.8 \mathrm{~m} / \mathrm{h}$ ) and the treated water flows in upward direction through the moving bed. This counter-current activity between water and sand ensures better treatment efficiency and effluent quality. But, the downward movement of sand, its distribution and actual sand circulation rate are often prone to deviations affecting the filtration efficiency. On-site and field surveys reveal that numerous $\mathrm{MBF}$ units operate at arbitrary circulation speeds and suffer from non-optimal operational conditions. They require every day monitoring to meet the stringent discharge limits, overcome malfunctions and reduce downtime. Sand-Cycle, thus functions as a continuous monitoring and control tool to provide real-time status updates and support MBF optimization by mimicking the sand movement with the help of LF tags. The key elements of the developed tool include LF tags (transponders) with transmission frequency of $128 \mathrm{kHz}$, remote sensing, cloud storage, big data analysis and expert judgement. The tags typically consist of a copper coil that is hermetically sealed in a bio-glass case designed to survive harsh environmental conditions (Figure 2). 

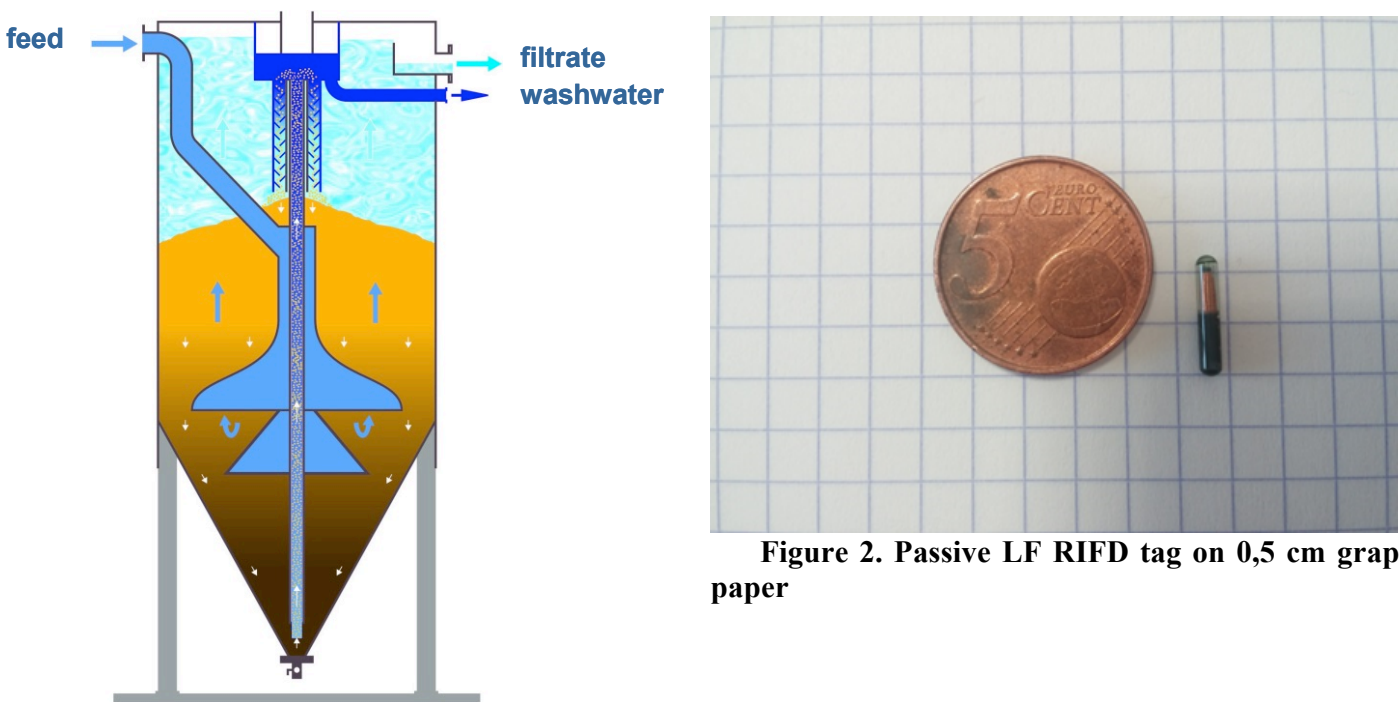

Figure 2. Passive LF RIFD tag on $0,5 \mathrm{~cm}$ graph paper

Figure 1. Schematic diagram of pollutant filtration through a sand bed using moving bed biofilters

The proof-of-concept of Sand-Cycle was carried out at pilot-scale in one filter of wastewater works of Franeker (Wouters, Heslegrave, Sutman, \& Opijnen, 2016). The health of the (bio)filters was visualized on a digital dashboard after translating the tags activity into performance grading factors such as sand circulation rate, active bed volume and filter homogeneity. Later, the smart monitoring tool has been extended to full scale application at various wastewater treatment facilities across EU. The ultimate goal is to increase reliability, reduce plant failures and improve overall treatment performance using this RFID-based technology. In the present article, a practical overview of long-term and full-scale implementation to track MBF's performance at two wastewater treatment plants (case-sites) is provided. System benchmarking was based on sand circulation rate, standard deviation, active bed volume and filter homogeneity. A concise discussion of the historical performance and observed fluctuations related to various aspects of filter operation is included. Furthermore, economic analysis was performed to illustrate the significance of smart and costeffective monitoring in day-to-day unit operations of wastewater treatment works.

\section{Background}

Two wastewater treatment works within EU region have been considered as case sites for this study viz. municipal wastewater treatment plant of Franeker (WwTW Franeker) and Anonymous sewage treatment works (WwTW A) of England. These two case sites were primarily selected among other sites as they handle similar wastewater load on daily basis, 14,400 and $15,861 \mathrm{~m}^{3} /$ day (max.) respectively. WwTW Franeker (Case site 1) is operated by Wetterskip Fryslan (a Dutch water authority) and is situated in the Frisian province of the Netherlands. The treatment cascade of case site 1 includes presettling $\rightarrow$ mineral filters $\rightarrow$ post settling which has been extended for simultaneous N-P removal via MBFs in 2008. The MBF plant consists of 6 concrete filter units, each equipped with 4 filter cells. Each filter unit is $5 \times 5 \mathrm{~m}$ with a bed height of $4 \mathrm{~m}$. The filter cells in each filter unit are 
not separated and hence each filter unit acts as an individual filter. The permissible limits of overall effluent are set at values of total- $\mathrm{N}(10 \mathrm{mg} / \mathrm{l}$, annual average $)$ and total-P $(2 \mathrm{mg} / \mathrm{l}$, rolling average in 10 consecutive daily samples). Ferric salt $\left(\mathrm{FeClSO}_{4}\right)$ and carbon-source (bio-ethanol) are dosed in the filter feed for effective phosphorus removal and denitrification. The dosages level of these chemicals is proportional to the feed flow and to actual feed NOx-N concentration and the actual feed oxygen concentration (for bio-ethanol) and inlet $\mathrm{PO}_{4}{ }^{3-}-\mathrm{P}$ values (for $\mathrm{FeClSO}_{4}$ ). On the other hand, case site 2 located in the Wiltshire English County operates under the administration of Thames Water Utilities Limited (a regulated Water and Sewerage Company). WwTW A employs conventional activated sludge systems with tertiary moving bed biofilters (NSF/MBF: sand filters) for effluent polishing. NSFs were introduced into the treatment configuration in the year of 2012 as 8 individual cylindrical MBFs of $2 \mathrm{~m}$ bed height placed in two rows. The consent levels for effluent discharge are set at finer values of ammonium-N (2.4 mg/1, 95\%ile) and average total-P $(0.8 \mathrm{mg} / 1,95 \%$ ile $)$. Important operational characteristics of the MBFs and Sand-Cycle setup on the two case sites are summarized in Table 1.

\begin{tabular}{lccc}
\hline Parameter & Case site 1 & Case site 2 & Unit \\
\hline Average Flowrate & 14,400 & 8,208 & $\mathrm{~m}^{3} / \mathrm{day}$ \\
Population equivalence & 60,000 & 10,800 & $\mathrm{P} . \mathrm{E}$ \\
$\begin{array}{l}\text { Type of MBF } \\
\text { Year of construction }\end{array}$ & AS500-40 & DS7000 DYNAOXYS & \\
$\begin{array}{l}\text { No. of filters } \\
\text { Average sand circulation }\end{array}$ & 2008 & 2012 & \\
rate & 4 & 8 & $\mathrm{~mm} / \mathrm{min}$ \\
Filter bed height & 4 & 6 & \\
$\begin{array}{l}\text { Surface area per filter } \\
\text { Bed volume per filter }\end{array}$ & 5 & 2 & $\mathrm{~m}^{2}$ \\
$\begin{array}{l}\text { Year of Sand-Cycle } \\
\text { integration }\end{array}$ & 2016 & 7 & $\mathrm{~m}^{3}$ \\
No. of transponders per filter & 100 & $19.5-20$ & \\
\hline
\end{tabular}

Table 1. Overview of the characteristics of moving bed biofilters and Sand-Cycle setup at Franeker Sewage Treatment Works (case 1) and Anonymous Sewage Treatment Works (case 2)

After integration of the real-time monitoring framework at case site 1 and case site 2, strategic optimization of the MBFs was carried out by the plant operators by (1) minimizing chemical dosage, (2) reducing energy consumption and (3) operating the filters at a practically low sand circulation rates. Lower sand (grain) circulation rates trigger better biological transformation of nitrates into nitrogen gas, removal of suspended solids and flocked phosphorus molecules. Continuous assessment of this sand circulation rate assisted in achieving an adequate process and indicated fluctuating and off-set conditions for immediate rectification.

\section{Methodology}

\subsection{Sand-Cycle tool}

The RFID tagging system primarily forms the core of the Sand-Cycle setup and includes the tags (transponders), a reading device (the reader) and a host system application for data collection, logging, processing and transmission. ID-1001H tags supplied by Trovan Ltd and DorsetID were used as identifiers in the tagging system. The tags are generally attached to the object that needs to be identified but in this case they mimic sand grains and are mixed up (50-100 tags per filter) together in 
the filter bed. They help in accounting of combined tag-sand grain circulation to monitor the filter operation. The tags are $11.5 \times 2.1 \mathrm{~mm}$ (length $\mathrm{x}$ diameter) in dimension and operate at a frequency of $128 \mathrm{kHz}$ (Dorset, 2017). Each tag (transponder) has a unique code at the time of manufacture which cannot be duplicated or tampered with. This code facilitates unique and positive identification as the transponder pass through the reader. Each transponder is detected while passing through the reader, which is integrated in the airlift structure of an MBF. The reader inductively energizes and excites the transponder by means of a polarized low frequency electromagnetic field emitted through an antenna. It then receives and processes the code signal bounced back by the transponder. Transponder signal can be read irrespective of their orientation with respect to the reader, also underwater (or in other fluids) and can be used in all weather conditions.

The codes, dates and time stamp of the passing transponders are then transmitted to a decoder that collects data from multiple readers. The decoder is connected to a data logger, which stores data received from the decoder in readable formats. The data logger is equipped with a GPRS modem to transmit the data to the back end of online data server. The data server is converting the raw field data into relevant output data using dedicated algorithms and big data analysis. Output is available 24/7 for the operators via the server's front office and is illustrated as digital dashboards.

\subsection{Data and filter performance analysis}

Large amounts of data points are generated by the 50-100 transponders which are translated into in-situ (near real time) filter performance grading factors using big data analytics. The dashboards present measurements, sand circulation $(\mathrm{mm} / \mathrm{min})$, active bed volume, last activity, 4-hour moving average sand circulation speed $(\mathrm{mm} / \mathrm{min})$ with standard deviation for defined time period, filter homogeneity and turnover per filter (case-specific). Measurements account the total transponder measurements since the start-up and sand circulation displays the in-situ 4-hour average sand circulation rate. The sand circulation is calculated by converting turnaround time of an individual transponder into speed and averaging it over 4-hour time period. This value depends on the characteristics of the filter used in operation. The time plot of sand circulation is represented as a profile of 4-hour moving average circulation speed indicating the overview of in-situ circulation rates. The spread of upper and lower asymmetrical standard deviation across the average over a defined period of time indicates the filter homogeneity level. Great/wider spread depicts non-distributed sand movement over the cross section of filter bed and lower performance level. Other grading factors such as active bed volume and turnover rate per filter are estimated based on the transponder detection over a fixed time period. They help in recognizing degree of filter bed stagnation, clogging, flush out of sand grains and migration of sand across filters. The combination of filter bed resistance and inflow rate with average sand circulation is functional as a controlling parameter for process optimization.

\subsection{Economic analysis}

Economic quantification of the consequences of filter plant malfunctioning was performed based on the results of surveys on MBF plants performed over the last two decades. Four important parameters like operator attendance, sand loss, energy needs and downtime have been shortlisted for economic analysis. The impact of in-situ and long-term monitoring using Sand-Cycle on these individual parameters was carried out to estimate the annual savings per parameter as a function of plant capacity ( $1 / \mathrm{s})$. Also, 25 -year lifetime net savings (in $€$ ) have been calculated after deducting the capital costs, maintenance and service charges for the hardware. 


\section{Results and discussion}

\subsection{Filter plant performance and off-set conditions}

Till date, the readers of each filter across the two case sites have transmitted about 10,000-21,500 signals from 50-100 transponders/filter cell to the decoder. These signals showed to be representative of the sand grain movement and better functioning of the hardware. Huge number of measurement points also promoted proper statistical analysis via data analytics and big data management for better understanding of the sand filtration. The results visualized on a dashboard web-interface provided insitu status of the filter to the operator.

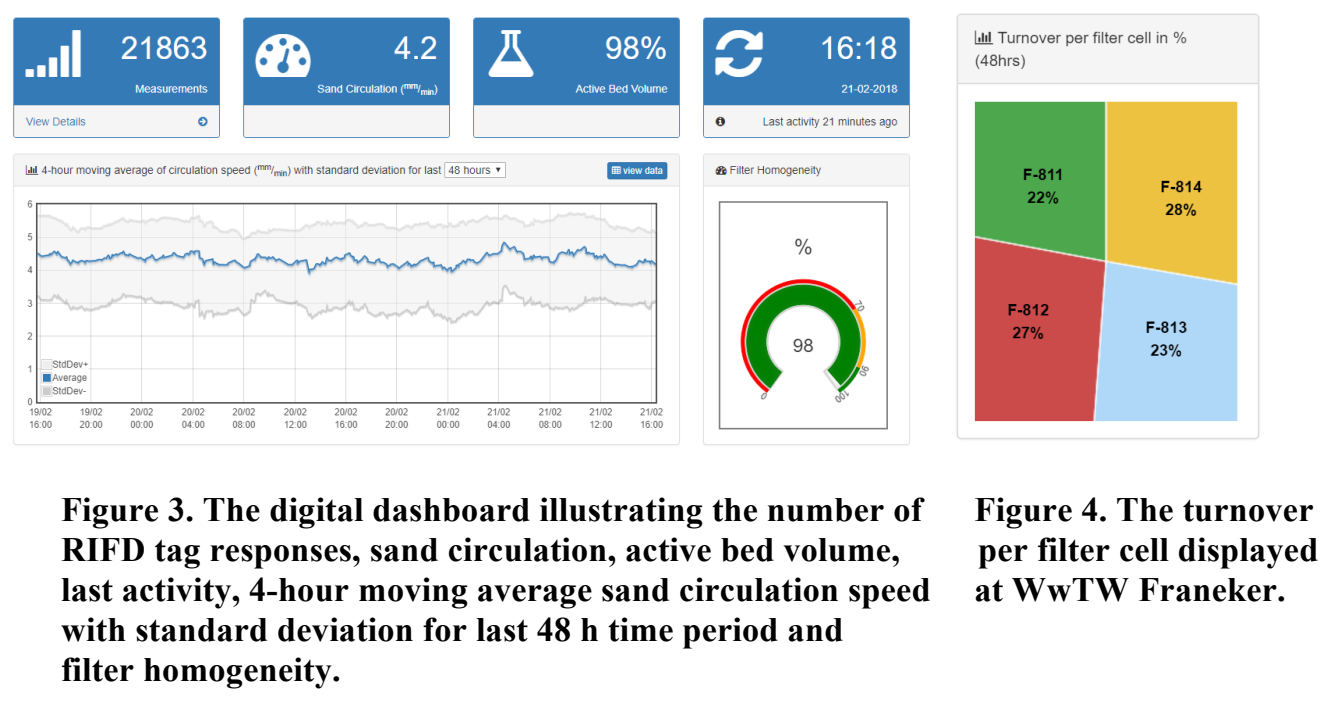

Figure 3 represents the complete dashboard as seen by the plant operator of WwTW Franeker (case site 1) to visualize the near real-time condition of a filter cell. The dashboard elements provided an overall impression of the filter status throughout the period of usage. Of these elements, the circulation rate and profile of circulation rates, upper and lower standard deviation represent the two key indicators to assess the filter health. The circulation rate also acts as the controlling parameter for optimizing removal efficiencies and biological conversions and can be fine-tuned to improve the filter performance. In case site 1, the filter activity looks in accordance with good performance. But, the sand was observed to migrate within the four cells of an individual filter unit during operation. This migration was visualized as percentage turnover for each cell (Figure 4) where the cells are unevenly distributed $(>$ or $<25 \%)$. Fine tuning of air flow per filter cell could promote homogeneous circulation and uniform distribution.

The four-month profile of circulation rates, upper and lower standard deviation of four filter cells at WwTW A (case site 2) indicated their sand bed movement over the cross-section of individual filter bed and corresponding homogeneity (Figure 4). Uniform distribution of upper and lower speed levels and low levels of deviation implies better homogeneity which eventually leads to better process performance. Higher deviations might correspond to filter anomalies such as stagnant zones. NSF - 1 and NSF - 5 had steady and uniform spread of speeds wheras NSF - 2 and NSF - 7 displayed a more non-uniform spread which corresponds to homogeneity values $<75-80 \%$. Non-homogenous sand circulation could be caused by various practical reasons. 
NSF - 1

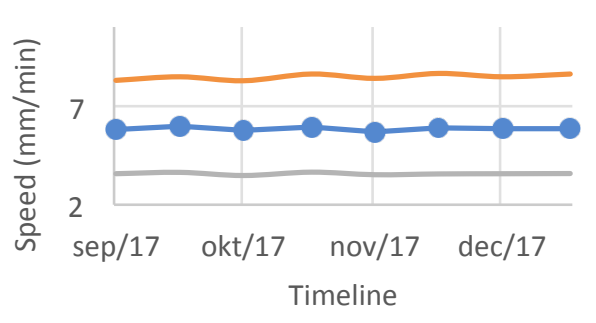

NSF - 5

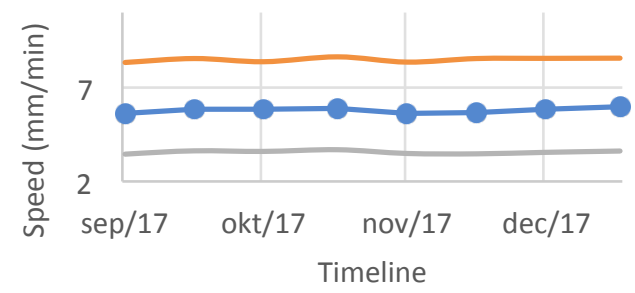

NSF - 2

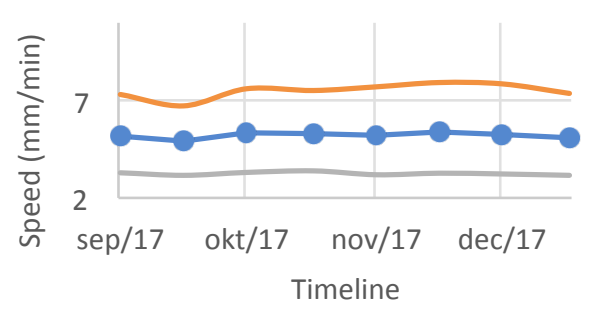

NSF - 7

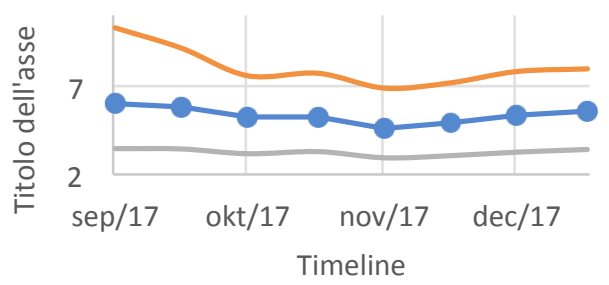

Figure 5. The profile of 4-hour moving average speed and standard deviation (in $\mathrm{mm} / \mathrm{min}$ ) of the four filters in WwTW A over Sept 2017 - Jan 2018. Red: upper standard deviation, orange: average speed and blue: lower standard deviation.

\subsection{Economic Analysis}

Big data analytics used in the case studies improved the equipment reliability and reduced the energy, maintenance and refurbishment costs. Identification of off-set conditions such as sand loss, downstream pipe blockages and filter bed stagnations via real-time status updates prevent deterioration of filter performance. Digital dashboards provide day-to-day monitoring of the filters and lessen the inspection time and operator attendance. The wireless transmission of operation warnings and diagnostics proved to be advantageous to operate the treatment unit at best possible conditions thereby assuring better effluent quality. For example, long-term observation of sand circulation could indicate the status of the airlift setup (wear and tear or energy requirements) and allows timely replacement. Figure 3 estimates the yearly and net 25 -year savings made on operator attendance, sand grain loss, energy consumption and downtime in relation to the plant capacity. Manpower costs can be immensely decreased for bigger plants which correspond to increasing trend of 25 -year net savings. The considerable cost reduction with respect to the assessed parameters estimates a return of investment of typically $1-2$ years. 


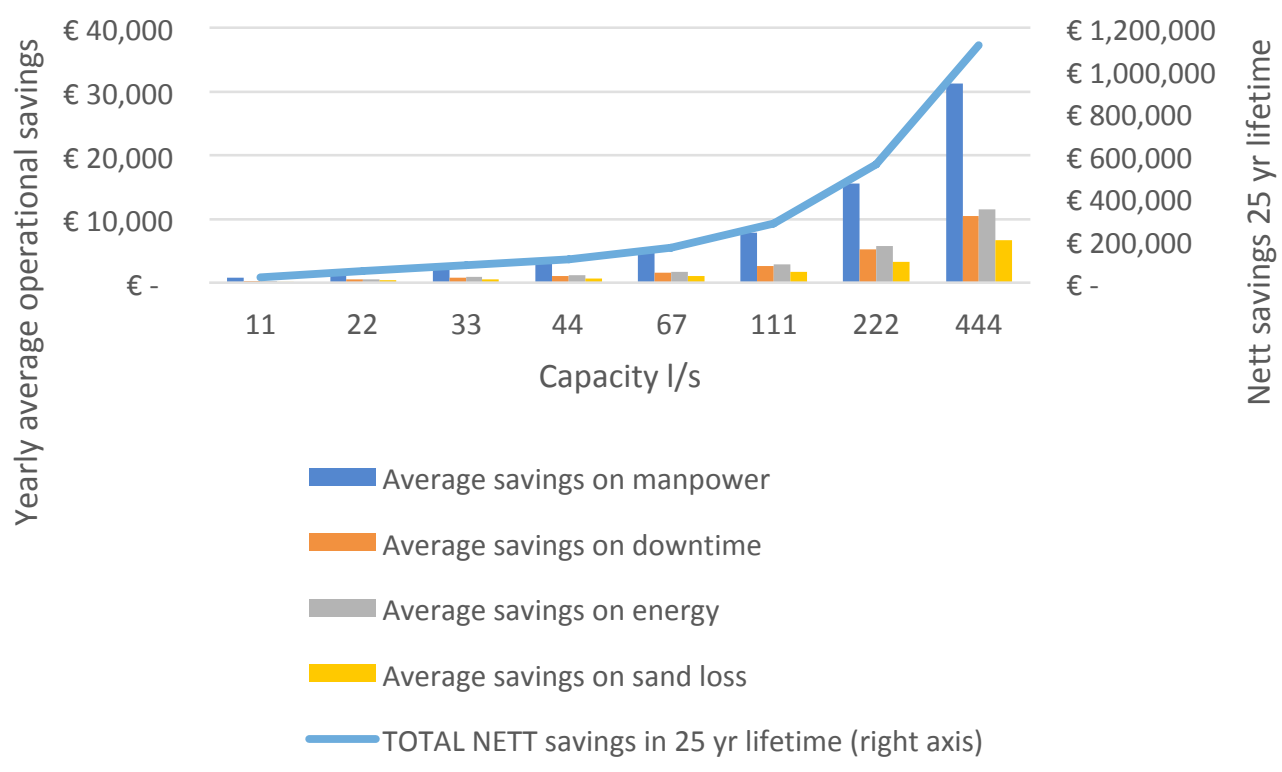

Figure 6. Estimated savings per parameter for 8 plant sizes based on their capacity (1/s).

\section{Conclusions}

Full-scale applications of a remote real-time tool for monitoring MBF plants has been successfully implemented at wastewater treatment works. Important results of the case studies reveal that:

- $\quad$ RFID technology is a promising, innovative and powerful tool for real time monitoring of MBF and can monitor overall plant performance. The results obtained at WwTW Franeker showed excellent reproducibility and added value to the plant operators.

- MBF plants equipped with Sand-Cycle are likely to have increased asset reliability and improved uptime percentages as early detection of dysfunctions and process disturbances allow the operators to reduce plant attendance without losing control.

- The return on investment of the tool, typically less than 2 years, allows the asset manager to justify the implementation of the technology.

- Introduction of similar advanced monitoring technologies (e.g. free flowing smart sensors) could pave path for effective and efficient wastewater management.

\section{References}

Dorset. (2017, October 26). Dorset. Retrieved from RFID Technology: http://www.dorset.nu/en/home-id-2/rfid_technology/.

F. Bibi, C. G. (2017). RFID technology having sensing aptitudes for food industry and their contribution to tracking and monitoring of food products. Trends Food Sci. Technol., vol. 62, pp. 91-103. 
J.P., K., J.W., W., M.P., N., \& J.M., A. D. (2000). Dynamic denitrification of $3600 \mathrm{~m} 3 / \mathrm{h}$ sewage effluent by moving bd biofiltration. Water Sci. Technol. vol. 41, no 4-5.

Leena Kumari, K. M. (2015). Application of RIFD in agri-food sector. Trends Food Sci. Technol. vol. 43 , no. 2, pp. 144-161.

SandCycle. (2017, October 26). Retrieved from Accelerate your filter performance and save costs: https://sand-cycle.com/

The EU Water Framework Directive. (2010).

Wouters, J., Heslegrave, C., Sutman, P., \& Opijnen, E. (2016). Making the most of continuously moving bed polishing (bio)filters for $\mathrm{N}$ and $\mathrm{P}$ removel, using sand-cycle remote control. 10th European Watewater Management Conference. AwuaEnviro.

X. Zhu, S. K. (2012). A review of RIFD technology and its managerial applications in different industries. J. Eng. Technol. Manag. - JET-M, vol. 29, no. 1, pp. 152-167. 